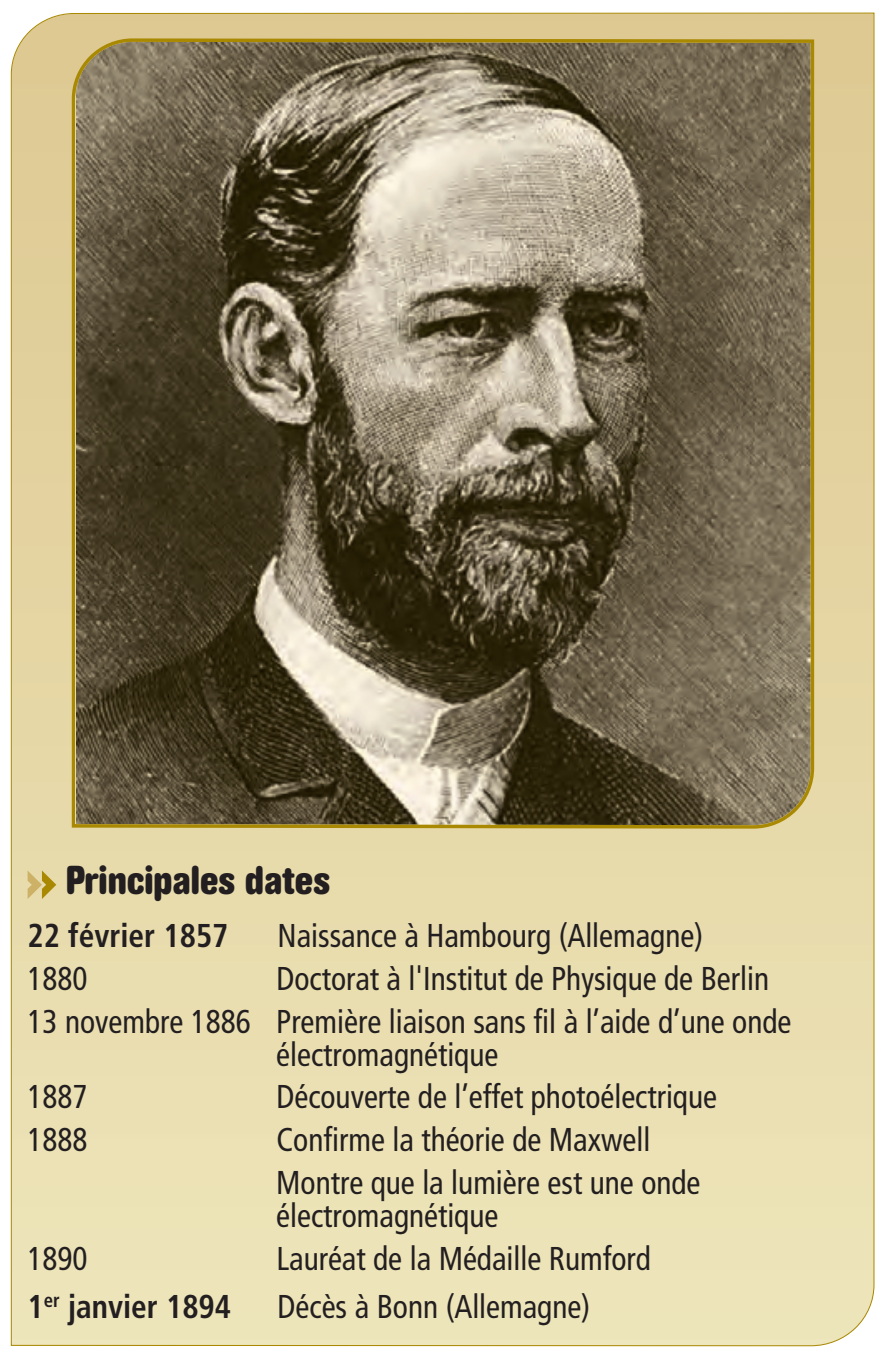

\title{
Heinrich Rudolf Hertz
}

Ingénieur et physicien allemand, Hertz est surtout connu pour avoir apporté la preuve expérimentale que la lumière est une onde électromagnétique qui obéit aux équations de Maxwell. En réalisant la première liaison sans fil entre un émetteur et un récepteur, il ouvre la voie à la télégraphie sans fil (inventée par Marconi) et à la radiophonie. II découvre en 1886 l'effet photoélectrique qui jouera un rôle central dans la théorie des quantas de lumière.

einrich Rudolf Hertz naît dans une famille aisée et cultivée He Hambourg le 22 février 1857. Sa mère, Anna Elisabeth Pfefferkorn, fille d'un médecin de Francfort, a grandi dans la tradition luthérienne. Son père, Gustav Ferdinand Hertz, est barrister (ou avocat plaidant) à Hambourg ; il est nommé conseiller à la cour d'appel, puis élu sénateur en 1887. Né juif, il se convertit au luthéranisme. Heinrich, comme ses quatre frères et sœur cadets, est donc éduqué dans la religion protestante.

Le jeune Heinrich entre, à six ans, à l'école privée dirigée d'une main de fer par le docteur Richard Lange, où le niveau d'enseignement est exigeant. Sous l'attention vigilante de sa mère, il s'avère un élève vif et studieux, particulièrement habile pour les activités manuelles, et notamment le travail du bois.

$\gg$

\section{Eulletın d'abunnement}

DÉSIGNATION

QUANTITÉ $\quad$ TOTAL

Abonnement (6 numéros)

$\square$ Tarif normal : $\square$ France : $70 €$ TTC $\bullet \square$ UE : $85 €$ TTC $\bullet \square$ Autres pays : $95 €$ TTC

$\square$ Étudiants (à titre individuel, sur justificatif) $\square$ France/UE : $45 €$ TTC $\bullet \square$ Autres pays : $65 €$ TTC

Commande de numéros déjà parus (10€ par exemplaire)

Indiquer ci-dessous le numéro de la revue commandée et, en dessous, la quantité souhaitée.

ex:

$$
\mathrm{PH} 4 \mathrm{O}
$$

$\mathrm{PH} 40 \mathrm{PH}$

$\mathrm{PH}$

$\mathrm{PH}$

$\mathrm{PH}$

$\mathrm{PH}$

$\mathrm{PH}$

$\mathrm{PH}$

3

TOTAL

$\square$ Je joins un chèque de

$\square$ Je règle par carte bancaire :

Date de fin de validité $L$ euros à l'ordre d'EDP Sciences

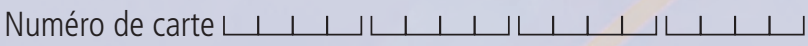

Cryptogramme visuel (Code inscrit au dos de ma carte)

$\square$ Je préfère régler par chèque, à réception de facture (uniquement entreprises et institutions)

$\square$ Merci de me faire parvenir une facture acquittée

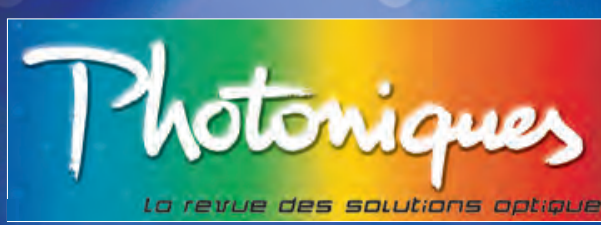

Photoniques $n^{\circ}$ 49/50 • novembre-décembre 2010 Photoniques $n^{\circ} 51 \quad$ - janvier - février 2011

Photoniques $n^{\circ} 52 \bullet$ mars-avril 2011

Photoniques $n^{\circ} 53 \quad$ - mai-juin 2011

Photoniques $n^{\circ} 54 \quad \bullet$ juillet-août 2011 Photoniques $n^{\circ} 55$ - septembre-octobre 2011

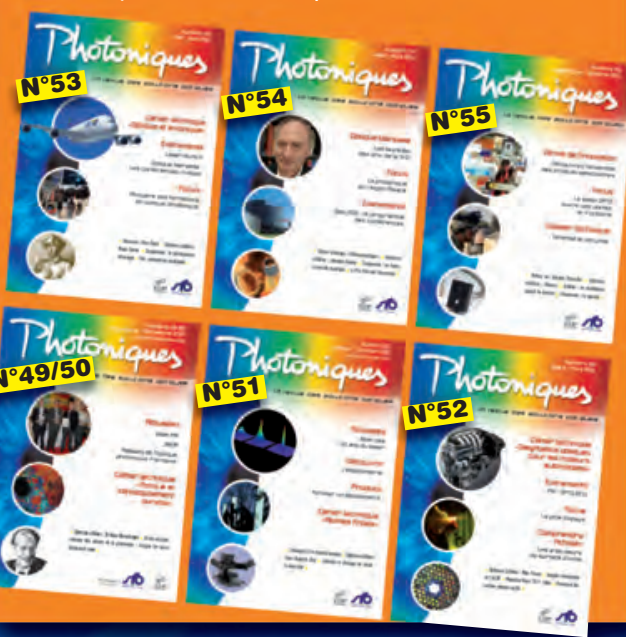




\section{QPTICIENS CÉLĖBRES}

\section{Education}

En 1872, il intègre le prestigieux Johanneum. II a alors 15 ans, et montre un intérêt prononcé pour les langues. Il étudie également le dessin technique sous la férule d'un professeur particulier. Il obtient, sans surprise, son Abitur avec mention en 1875. Doué pour à peu près tout, il doit trancher entre deux cursus: celui, à forte dominante théorique, qui ouvre sur une carrière académique ou celui, nettement plus appliqué, qui mène à l'industrie. II opte après mûre réflexion pour un cycle d'ingénieur en BTP, et s'inscrit à Francfort. Il y suit une formation pratique, et se prépare aux concours d'état.

Mais très vite, le rythme et l'environnement de travail du secteur privé lui pèsent. Il se sent à l'étroit, enfermé dans une aventure individuelle. Il se prend à penser qu'il a choisi le mauvais cursus. Pour autant, il hésite à l'interrompre et à perdre le temps déjà investi. Toujours indécis, il s'inscrit en 1876 à la KöniglichSächsisches Polytechnikum de Dresde. Mais il n'y reste qu'une courte période, avant $d$ 'intégrer le régiment du rail pour un service militaire d'un an. Puis, reprise du cycle d'ingénieur...

Il faut cependant que ce jeu de yoyo cesse. Hertz sait désormais qu'il veut, par-dessus tout, mener une existence de chercheur scientifique, symbole pour lui d'un idéal d'apprentissage et de savoir. Il lui reste à convaincre son père, qui finance ses études, de le soutenir dans son projet de réorientation. Retour à Hambourg et plaidoyer enflammé auprès du barrister qui, convaincu par sa ferveur et son enthousiasme, valide le choix de son fils.

Hertz s'inscrit à la München Universität en 1877 et se met à jour en mathématiques. Son professeur Von Jolly [1809-1884] lui conseille la lecture de Lagrange, Laplace et Poisson... La beauté extraordinairement efficace des mathématiques le séduit immé- diatement, mais Hertz est déjà intimement, et restera tout au long de sa carrière, un physicien convaincu.

\section{Nouveau départ}

L'année suivante il s'inscrit à Berlin, où il suit les cours de Helmholtz [1821-1894] et Kirchhoff [1824-1887]. C'est une révélation, qui confirme qu'il est désormais dans le bon cursus. Il veut s'impliquer sans attendre dans un projet de recherche, et décide de concourir au prix de la Faculté de Philosophie pour l'étude expérimentale de l'inertie électrique. Helmholtz perçoit l'enthousiasme, en même temps que l'énorme potentiel technique et scientifique du jeune homme, et accepte de l'introniser : il I'héberge dans une salle de I'Institut de Physique, et le guide dans ses recherches bibliographiques... Sous la férule attentive de ce maître de la physique, Hertz fait un travail remarquable et remporte la médaille d'or en 1879.

Helmholtz lui propose alors, de concourir au prix de l'Académie des Sciences de Berlin en concevant une expérience permettant de vérifier la théorie de Maxwell [1831-1879] selon laquelle la lumière serait une onde électromagnétique. Hertz est tenté mais ne se sent pas prêt à s'embarquer dans un projet de plusieurs années et souhaite plutôt passer un doctorat : il rédige en quelques mois une étude théorique de l'induction, Über die Induction in rotirenden Kugeln, qu'il soutient publiquement en février 1880. Il devient ensuite pendant trois années très productives I'assistant de Helmholtz. II publie beaucoup, et propose quelques innovations comme de nouveaux ampère-mètre et hygromètre. En 1883, désireux de faire progresser sa carrière, mais intimidé par la rude concurrence locale, Hertz décide de quitter le havre protecteur de l'Institut de Physique et la proximité stimulante

\section{$\square$ Monsieur}

$\square$ Madame

$\square$ Mademoiselle Société

Adresse complète (précisez BP, Cedex...)

Code postal

Téléphone

E-mail

Pour que nous puissions adapter Photoniques aux besoins de ses lecteurs, merci de bien vouloir nous indiquer

\section{Votre service :}

$\square$ Direction générale

$\square$ Conseil. Marketing. Publicité

$\square$ Études. Développement

$\square$ Méthodes. Ordonnancement

$\square$ Recherche

$\square$ Contrôle

$\square$ Production

$\square$ Entretien. Sécurité

$\square$ Achats

$\square$ Enseignement. Formation

$\square$ Autre :

\section{Votre fonction :}

$\square$ Chef d'entreprise. DG

$\square$ Directeur

$\square$ Chef de service

$\square$ Ingénieur

$\square$ Chef de projet

$\square$ Technicien

$\square$ Agent de maîtrise

$\square$ Chercheur

$\square$ Directeur de recherche

$\square$ Universitaire (enseignant, chercheur)

$\square$ Autre
Prénom

Service..
Pays

Fax.

........................ 
de son mentor. Bien qu'expérimentateur dans l'âme, il se porte candidat à une position de privatdozent en physique théorique à la Christian-Albrechts-Universität de Kiel. Puissamment parrainé par Helmholtz, il obtient le poste sans difficulté.

\section{L'enseignement}

En quittant Berlin pour Kiel, Hertz perd l'accès aux formidables moyens techniques et technologiques de I'Institut de Physique. Le sevrage expérimental est rude. Mais Hertz a également plus de temps libre et il donne toute la mesure de son talent d'enseignant. En 1884, il fait sa première incursion dans la théorie de Maxwell et les ondes électromagnétiques : il publie un papier qui marque sa conversion aux concepts de la théorie des champs; c'est un vrai changement de paradigme.

Mais il ne reste que deux ans à Kiel. En 1885, il accepte un poste à la Technische Hochschule de Karlsruhe, comme assistant du futur Prix Nobel Karl Ferdinand Braun [1850-1918]. Sa vie privée prend un tournant : il fréquente Elizabeth Doll, la fille d'un collègue, et l'épouse en juillet 1886. Ils auront deux enfants, Johanna et Mathilde.

Après une période de réflexion, longue et parfois pénible, Hertz choisit de traiter (enfin !) le problème que lui a soufflé Helmholtz sept ans plus tôt : vérifier par l'expérience la théorie de Maxwell, selon laquelle la lumière est une onde électromagnétique. Par bien des aspects, ce travail se place dans la continuité de son papier théorique de 1884. Chronique d'un formidable tour de force scientifique : le 13 novembre 1886, il réalise la première liaison sans fil à l'aide d'ondes électromagnétiques dans l'air ; il montre que la vitesse de ces ondes est celle de la lumière ; il établit qu'elles obéissent aux lois de la réflexion et de la réfraction; il montre enfin, en 1888, que la lumière est ellemême une onde électromagnétique décrite par les équations de Maxwell. En 1887, il découvre également l'effet photoélectrique, par lequel une plaque de métal éclairée émet des électrons - une découverte qui jouera un rôle central dans la théorie des quantas de lumière d'Einstein.
Mais la portée de ses travaux lui échappe. S'il a révolutionné notre perception de la théorie de Maxwell, il n'a pas su prévoir les applications de ses découvertes; c'est en effet au génie pratique de Marconi [1874-1937] et de Braun que l'on doit la télégraphie sans fil.

\section{Ultima verba}

À 31 ans à peine, sa notoriété est telle que les offres de chaire affluent de toute l'Allemagne : Giessen, la grande ville universitaire du Land de Hesse ; Berlin, en remplacement de Kirchhoff; et enfin Bonn, à la chaire de Clausius [1822-1888]. En décembre 1888 , il cède au charme de Bonn, et d'une charge légère qui lui garantit le temps de réfléchir. II approfondit la théorie de Maxwell dans deux papiers, et projette une clarté conceptuelle inédite et particulièrement féconde sur la propagation des ondes électromagnétiques. Ses travaux lui valent la prestigieuse médaille Rumford de la Royal Society de Londres en 1890.

Depuis quelques années, Hertz souffre de sérieux problèmes de santé. Une douleur persistante aux dents et dans tout le système ORL, présente dès 1888, atteint en 1892 un niveau tel qu'il doit interrompre toute activité. Un traitement particulièrement lourd lui apporte un soulagement passager en 1893. Il reprend alors courageusement ses cours et achève le manuscrit de son livre Die Prinzipien der Mechanik qu'il envoie à son éditeur le 3 décembre. Mais une récidive le foudroie peu après : il décède

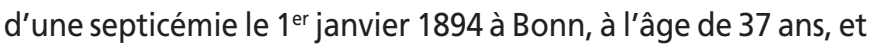
est inhumé à Hambourg, sa ville natale.

\section{$>$ Riad HAIDAR \\ Onera \\ haidar@onera.fr}

\section{Références}

Charles Susskind, « Heinrich Hertz : a Short Life ». San Francisco Press (1995).

\section{SPECTROGON}

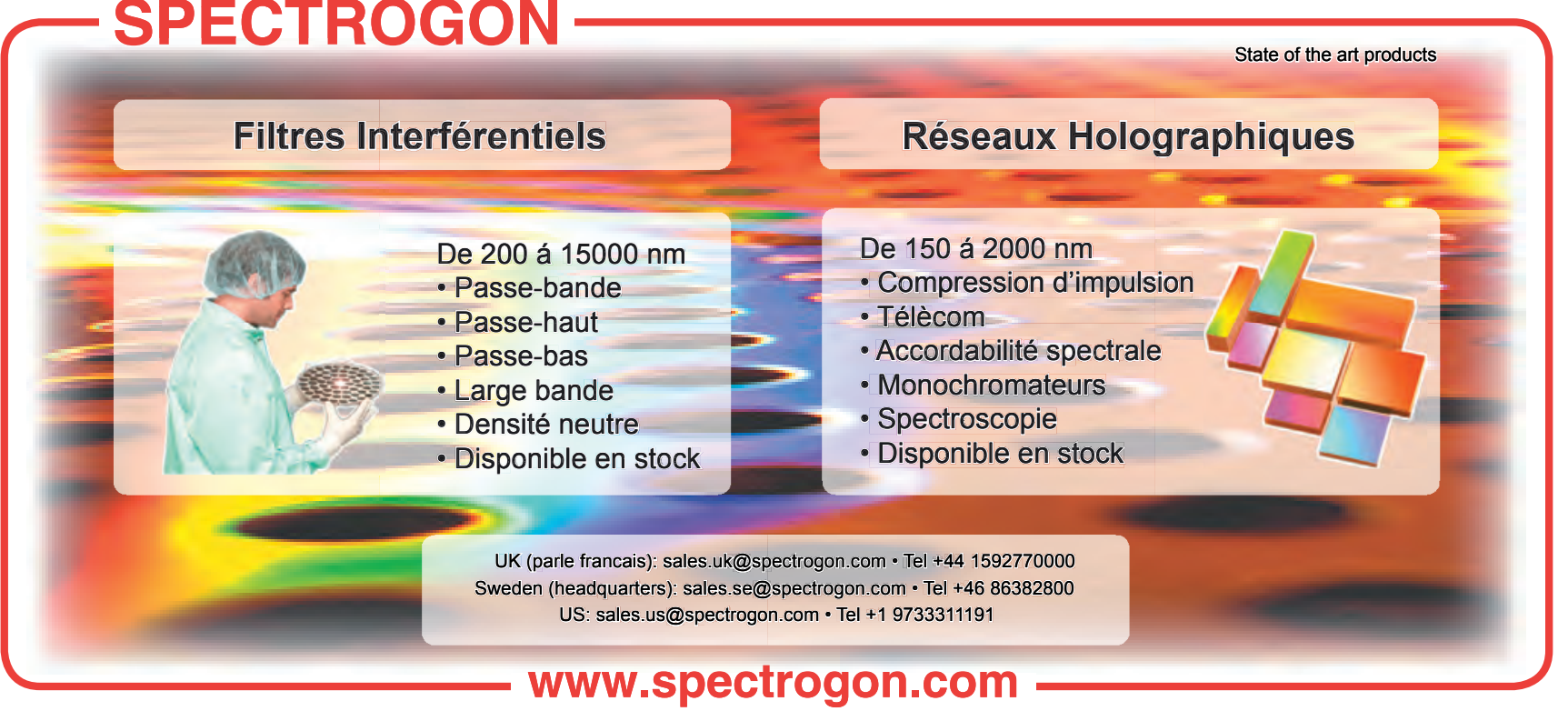

